\title{
Throughput-Delay Analysis of a Multichannel Wireless Access Protocol
}

\author{
A. Chockalingam, Senior Member, IEEE, Weiping Xu, Member, IEEE, Michele Zorzi, Senior Member, IEEE, \\ and Laurence B. Milstein, Fellow, IEEE
}

\begin{abstract}
In this paper, we analyze the effect of bursty packet losses, caused by a correlation in the multipath fading process, on the throughput and delay performance of a multichannel wireless access protocol. $M$ equal-capacity, orthogonal, traffic channels are shared by $N$ mobile users $(N \geq M)$ on the uplink (mobile-to-base station link). Transmission attempts on the uplink are made based on the busy/idle status of the $M$ receivers, which is broadcast by the base station, in every slot, on the downlink (base station-to-mobile link). Following a Markov chain analysis, we derive the analytical expressions for the average per channel throughput and the mean message transfer delay. Simulation results are shown to verify the analysis. We compare the effect of Doppler bandwidth on the performance of both the basic protocol without link layer error recovery and a modified version of the protocol that employs a "persist-until-success" link layer retransmission strategy to recover erroneous data packets.
\end{abstract}

Index Terms-Mobile communications, multipath fading, wireless access protocols.

\section{INTRODUCTION}

$\mathbf{N}$ EXT-GENERATION wireless networks are envisaged to support high data rates, packet-oriented transport, and multimedia traffic. The design of efficient and robust wireless media access protocols and the evaluation of their performance in the presence of physical layer impairments like channel fading are key technical issues [1]-[6]. High-capacity wireless networks may be realized either by assigning a single wide-band channel or by using multiple narrow-band channels that are orthogonal to each other. The latter approach, which we will consider in this paper, is particularly attractive when contiguous wide-bandwidth spectrum is not available. Orthogonal multiple narrow-band channels can be achieved, for example, in frequency, time, or code domains. In a multichannel system, there are several independent orthogonal channels, and a user can transmit on

Manuscript received April 15, 1997; revised April 7, 1999. This work was supported in part by TRW, Airtouch, the Center for Wireless Communications at the University of California, San Diego, and the MICRO Program of the State of California. This paper was presented in part at the IEEE International Conference on Universal Personal Communications, San Diego, CA, October 1997.

A. Chockalingam is with the Department of Electrical Communication Engineering, Indian Institute of Science, Bangalore 560012, India.

W. Xu is with Nokia Mobile Phones, Inc., San Diego, CA 92121 USA.

M. Zorzi is with the Dipartimento di Ingegneria, Università di Ferrara, 44100 Ferrara, Italy (e-mail: zorzi@ing.unife.it).

L. B. Milstein is with the Department of Electrical and Computer Engineering, University of California at San Diego, La Jolla, CA 92093 USA.

Publisher Item Identifier S 0018-9545(00)02557-3. any of these channels based on a suitable access protocol. The performance of multichannel slotted ALOHA systems, where multiple equal-capacity channels are shared by many users, has been analyzed in [7] and [8]. However, these studies assumed a deterministic channel model that did not consider the effect of multipath fading on the system performance. In fact, mobile radio channels are severely affected by time-varying losses due to distance, shadowing, and multipath fading. While the variation in the losses due to distance and shadowing is relatively slow, the variation due to multipath fading is quite rapid [9], [10]. The fading envelope due to multipath typically follows a Rayleigh distribution whose autocorrelation function depends on the normalized Doppler bandwidth, which in turn varies as a function of the mobile user velocity [10]. In this paper, we analyze the throughput and delay performance of a multichannel wireless access protocol taking physical layer impairments into account. Specifically, we analyze the effect of bursty packet losses, caused by correlation in the multipath fading process, on the protocol performance.

In [11], we presented and analyzed a single channel wireless access protocol that makes use of the uplink (mobile-to-base station link) channel status information, which is conveyed to the mobiles through a busy/idle flag broadcast on the downlink (base station-to-mobile link). This protocol can be viewed as a hybrid protocol employing the slotted ALOHA and reservation concepts [12]. A header packet is sent on a contention basis first, following which data packets are sent on a reservation basis. By this approach, packet losses due to collision are restricted to occur only among header packet transmissions. In this paper, we extend this protocol to utilize multiple, orthogonal, traffic channels on the uplink [13]. The multichannel wireless access protocol is described in Section II. The analysis in [13] assumed the multipath Rayleigh fading to be independent and identically distributed (i.i.d.). However, the multipath fading process in mobile radio environments can typically be considered to be slowly varying, for the usual values of the carrier frequency (e.g., 900-1800 MHz) and for typical mobile speeds [10]. Particularly, the correlation in multipath fading introduces burstiness (memory) in the packet error process, which can affect the protocol performance. In Section III, we describe the fading channel model we use to analyze the protocol performance. In Section IV, we present the throughput-delay analysis of the multichannel protocol, taking into account the correlation in the fading process. We also analyze the performance of the multichannel protocol with a "persist-until-success" link level (LL) retransmission strategy to recover erroneous data packets. Section V provides the analytical and simulation re- 
sults. Conclusions and areas of future work are provided in Section VI.

\section{Multichannel Wireless Access Protocol}

Consider a packet communication wireless network where $N$ mobile users share $M$ equal-capacity, orthogonal, traffic channels $(N \geq M)$ on the uplink to communicate with the base station. All the uplink channels are synchronized and slotted to one packet duration. All the mobiles in the network can use any of the $M$ different channels following the access rules. The base station is provided with $M$ receivers to demodulate all the uplink channels' traffic. Based on the busy/idle status of the $M$ receivers, the base station broadcasts an $M$-bit busy/idle word, in every slot, on the downlink. The busy/idle flag corresponding to each channel is set or reset depending on whether or not the data packets are being transmitted on that channel.

Each message generated at the mobiles consists of two segments, namely the header segment and the data segment. The header segment is of one packet length. It carries control information, e.g., the destination address and the number of packets in the data segment. The data segment, which represents the actual traffic, consists of a random number of packets. Transmission attempts are made by the mobiles only at the slot boundaries by sending the header packet.

The mobile, once it receives a message to be sent to the base station, first checks the status of the busy/idle word which it periodically receives from the base station on the downlink. If all the $M$ busy/idle flags indicate busy status, the mobile refrains from making a transmission attempt and reschedules the attempt to a later time. If one or more flags indicate an idle status, then the mobile randomly picks one of these idle channels, and makes a transmission attempt by sending a header packet on the uplink slot of the chosen channel. If the header packet is received successfully, without packet loss due to fading or collision, the base station broadcasts the channel ID and the successful mobile ID (capturing mobile in the event of collision among header packets from different mobiles) and sets the corresponding channel's flag busy for the $X$ subsequent slots, where $X$ is the number of packets in the data segment of the successful mobile. This allows only the successful mobile to send its data packets in those $X$ slots on that channel. During these $X$ slots, other mobiles would receive a busy status flag for this channel and so they would not make transmission attempts on it. The base station resets the flag back to idle status after $X$ slots. If the header packet is lost (due to collision or fading), then the base station will not respond with a busy status flag, but will continue to send the corresponding channel's flag as idle. This is an indication to the mobile that the header packet was lost, and so it has to reschedule its transmission attempt to a later time.

Note that, when the feedback is error free, packet transmissions can experience fading, interference and noise during header slots, whereas data slots experience only fading and noise (no interference). Thus, in the case of error-free feedback to all the mobiles, collisions and hence capture are possible only during the header packet transmission and not during the transmission of data packets. However, errors in the busy/idle word reception will result in collisions, and hence packet losses, during the transmission of data packets as well.
TABLE I

MARKOV PARAMETERS AT DIFFERENT VALUES OF $f_{D} T$ FOR A TARGET ERROR RATE OF 0.0952 (i.e, FADING MARGIN OF $10 \mathrm{~dB})$. BPSK MODULATION WITH $(511,250,31)$ BCH CODE

\begin{tabular}{|c|c|c|c|c|c|c|}
\hline \multirow{2}{*}{$f_{D} T$} & Model & Avg SNR (dB) & $P_{E}$ & $p$ & $q$ & $(1-q)^{-1}$ \\
& & & & & & \\
\hline \hline 0.01 & Const. $S N R_{0}$ & 10.5 & 0.0963 & 0.9899 & 0.9056 & 10.59 \\
& Const. $P_{E}$ & 10.5 & 0.0963 & 0.9899 & 0.9056 & 10.59 \\
\hline 0.02 & Const. $S N R_{0}$ & 10.5 & 0.0954 & 0.9834 & 0.8426 & 6.35 \\
& Const. $P_{E}$ & 10.5 & 0.0954 & 0.9834 & 0.8426 & 6.35 \\
\hline 0.04 & Const. $S N R_{0}$ & 10.5 & 0.0958 & 0.9695 & 0.7122 & 3.47 \\
& Const. $P_{E}$ & 10.5 & 0.0958 & 0.9695 & 0.7122 & 3.47 \\
\hline 0.08 & Const. $S N R_{0}$ & 10.5 & 0.1009 & 0.9433 & 0.4950 & 1.98 \\
& Const. $P_{E}$ & 10.5 & 0.1009 & 0.9433 & 0.4950 & .198 \\
\hline 0.1 & Const. $S N R_{0}$ & 10.5 & 0.1037 & 0.9324 & 0.4159 & 1.71 \\
& Const. $P_{E}$ & 10.7 & 0.0994 & 0.9345 & 0.4068 & 1.68 \\
\hline 0.2 & Const. $S N R_{0}$ & 10.5 & 0.1149 & 0.8969 & 0.2066 & 1.26 \\
& Const. $P_{E}$ & 11.2 & 0.0991 & 0.9101 & 0.1831 & 1.22 \\
\hline 0.4 & Const. $S N R_{0}$ & 10.5 & 0.1059 & 0.9022 & 0.1751 & 1.21 \\
& Const. $P_{E}$ & 10.7 & 0.0998 & 0.9079 & 0.1698 & 1.20 \\
\hline
\end{tabular}

\section{A. Protocol with Link Level Retransmission}

In the basic multichannel protocol described previously, packets which get corrupted during the data segment transmission are lost and the recovery of such errors is left to the higher layer protocols. A simple way of recovering such data packet errors is through retransmission at the link level (LL) itself. Instead of ignoring packet errors, a data packet is retransmitted if it is received in error. In local wireless environments where the feedback delays are very small compared to the slot duration, a data packet in error can be retransmitted in the immediately following slot. Retransmission can persist until the packet is successfully received. In this case, the base station would need to send a nonbinary feedback (busy/idle/retransmit) in order to avoid a collision among retransmission packets from a mobile with header packets from other mobiles. We refer to this modified protocol as the protocol with "persist-until-success" LL retransmission.

\section{MARKOV CHANNEL MODEL}

In this section, we describe the fading channel model we use to analyze the performance of the protocols described above. We assume that all the mobiles' transmissions are power controlled so that the slowly varying distance and shadow losses are perfectly compensated, whereas the rapidly varying multipath fading remains uncompensated. We use a Markov channel model to characterize the correlated multipath fading, which is described in the following.

We consider the multipath fading to be frequency nonselective, which may be a reasonable assumption for data rates of the order of $100 \mathrm{kbps}$ or less as considered in this paper. The effect of fading is then described as a multiplicative complex function, $\alpha(t)$, whose envelope is assumed to have a Rayleigh distribution [10]. In [14], the packet success/failure process was modeled as the outcome of a comparison of the instantaneous signal-to-noise ratio to a threshold value, $\mathrm{SNR}_{t}$ : if the received SNR is above the threshold, the packet is successfully decoded with probability 1 ; otherwise, it is lost with probability 1 . If $F$ 
is the value of the fading margin of the link, the instantaneous signal-to-noise ratio (taking into account the effect of fading) is given by $\mathrm{SNR}_{0}|\alpha(t)|^{2}$, where $\mathrm{SNR}_{0}=\mathrm{SNR}_{t} F$ is the average value of the SNR at the receiver. Hence, the binary process that describes packet successes/failures on the channel $\left(\beta_{i}\right)$ can be obtained by comparison of the squared magnitude of $\alpha(t)$ with the threshold $1 / F$. This gives rise to a binary packet error process which can be well approximated by a two-state Markov model with transition matrix

$$
\mathbf{M}_{\mathbf{c}}=\left(\begin{array}{cc}
p & 1-q \\
1-p & q
\end{array}\right)
$$

where $p$ and $1-q$ are the probabilities that the packet transmission in slot $j$ is successful, given that the packet transmission in slot $j-1$ was successful or unsuccessful, respectively. ${ }^{1}$ The steady-state probability that a packet error occurs $\left(P_{E}\right)$ is [14]

$$
P_{E}=\frac{1-p}{2-p-q}
$$

For given values of the fading margin $(F)$ and of the Doppler frequency $\left(f_{D}\right)$ normalized by the packet transmission rate $(1 / T)$, we have [14]

$$
P_{E}=1-e^{-1 / F}
$$

and

$$
q=1-\frac{Q(\theta, \rho \theta)-Q(\rho \theta, \theta)}{e^{1 / F}-1}
$$

where

$$
\theta=\sqrt{\frac{2 / F}{1-\rho^{2}}}
$$

$\rho=E\left[\alpha(t) \alpha^{*}(t+T)\right]=J_{0}\left(2 \pi f_{D} T\right)$ is the correlation coefficient of two samples of the complex amplitude of the fading process taken $T$ s apart [9], [10], $J_{0}(\cdot)$ is the Bessel function of the first kind and of zeroth order, and $Q(\cdot, \cdot)$ is the Marcum $Q$ function, defined as

$$
Q(z)=\int_{z}^{\infty} \frac{1}{\sqrt{2 \pi}} e^{-y^{2} / 2} d y
$$

Equations (3) and (4), together with (2), provide the complete statistical characterization of the packet error model in this case.

The derivation of this threshold model implicitly assumes that the fading envelope does not change during a packet and that the relationship between SNR and packet error probability is a step function. However, it has been shown in [15] that more realistic systems can still be modeled in this way, by defining an appropriate equivalent fading margin, which depends on the physical layer details (coding and modulation as well as the average signal-to-noise ratio, $\mathrm{SNR}_{0}$ ). The parameters of the

${ }^{1}$ Following the established use for Markov chains, we arrange all transition probabilities from the same state in a row, i.e., $M_{i j}$ is the probability of a transition from $i$ to $j$.
Markov model can in this case be evaluated by simulation, as explained in [15].

When considering different values of the Doppler frequency, we can follow two approaches. In the first approach, we let $f_{D} T$ vary while keeping constant all physical layer parameters, including $\mathrm{SNR}_{0}$. The average packet error rate $\left(P_{E}\right)$, which is relatively constant for small values of $f_{D} T$, will eventually show some dependence on $f_{D} T$ as $f_{D} T$ itself increases (for example, when coding is used, faster fading will eventually produce better packet error rate performance due to low correlation among bits in the same codeword). This comparison (for constant $\mathrm{SNR}_{0}$ ) can be useful when assessing, for example, the effect of the mobile speed on the system performance without any control on the packet error rate.

In the second approach, we assume that for all values of $f_{D} T$ the packet error rate $\left(P_{E}\right)$ is held constant. This means that, for some values of $f_{D} T$, we need to adjust the transmit power in order to achieve the target value of $P_{E}$. This approach is useful in accounting for correlation effects in the packet error modeling, since the case of large $f_{D} T$ corresponds to completely neglecting the second-order fading statistics. Also, this approach may correspond to a real system implementation where the physical layer includes a power control algorithm which tries to maintain the packet error rate at some target value, regardless of the fading bandwidth. For example, the overall power control strategy can be based on estimates of the packet error rate rather than on attenuation measurements.

In discussing the numerical results, we will consider both approaches. Note that in the latter approach, we can use the threshold model without worrying about the physical layer details, since we can use a constant value of the fading margin for all values of $f_{D} T$ [note from (2) that in the threshold model a constant $P_{E}$ is equivalent to a constant $F$ ]. In the first approach, on the other hand, we need to specify the physical layer parameters of the scheme, in order to be able to track the variations of the Markov model as a function of $f_{D} T$. As a concrete example, in this paper we will consider the case in which each block is a codeword of a rate- $1 / 2,(511,250,31) \mathrm{BCH}$ code, which can correct up to 31 bits per block [16]. The value of $\mathrm{SNR}_{0}$ will be chosen such that for slow fading the packet error rate is the same as for the corresponding threshold model. Table I shows the Markov parameters at different values of $f_{D} T$ for a target error rae of 0.0952 (i.e., 10-dB fading margin), obtained through the technique outlined in [15] for the above BCH code with BPSK modulation, both in constant $\mathrm{SNR}_{0}$ (first approch) and constant $P_{E}$ (second approach) conditions.

\section{PERFormance AnAlysis}

In this section, we analyze the throughput and delay performance of the multichannel wireless access protocol described in Section II. A single-cell system with no intercell interference is considered. In order to analyze the system, we assume that the busy/idle word on the downlink is received instantaneously, and error free by all the mobiles. In practice, the busy/idle word might be corrupted by the channel (a busy status being flipped to idle status and vice versa). The reliability of the busy/idle word reception could be enhanced by providing adequate error protection. The instantaneous feedback assumption is appropriate 


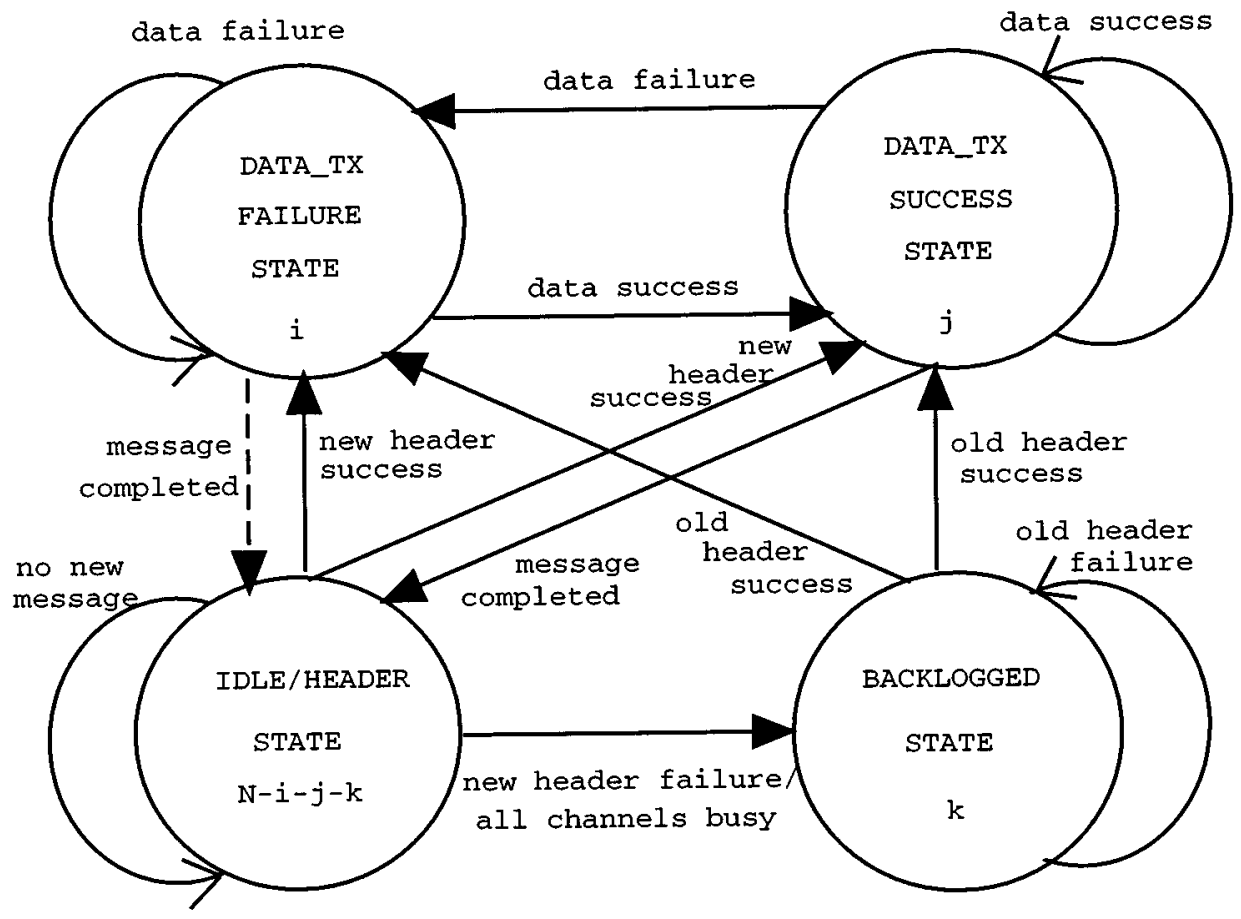

Fig. 1. Mobile state transition diagram (in Markov fading).

where the delays due to propagation and processing are small compared to the slot duration.

A new message is assumed to arrive at each mobile with probability $\lambda$ in each slot (Bernoulli arrival process). The mobile accepts a newly arriving message for transmission only when it has no current message to send and does not generate new messages when it already has a message to send. The length of the data segment of each message $(X)$, measured in integer number of packets, is assumed to follow a geometric distribution with parameter $g_{d}$, and probability mass function

$$
P[X=i]= \begin{cases}g_{d}\left(1-g_{d}\right)^{i-1}, & i=1,2,3, \ldots \\ 0, & \text { otherwise }\end{cases}
$$

As per the multichannel protocol described in Section II, in any given slot, each mobile can be in any one of four states, namely, idle/header_tx state, data_tx_success state, data_tx_failure state, and backlogged state (see Fig. 1). The data segment transmission is represented by separate success and failure states in order to take into account the one slot channel memory (defined by a first-order Markov chain with parameters $p$ and $q$ ). In the idle/header_tx state, the mobile remains idle with probability $1-\lambda$ or generates a new message with probability $\lambda$. In the latter case, the mobile randomly chooses an idle uplink channel (if available) and transmits the header packet in the uplink slot. If the header packet transmission is successful, the mobile moves from the idle/header_tx state to the transmission of data packets, i.e, to either data_tx_success state or data_tx_failure state. During the transmission of data packets, the mobile moves from data_tx_success state to data_tx_success state or data_tx_failure state with probability $p$ and $1-p$, respectively. Similarly, from data_tx_failure state, the mobile moves to data_tx_success state or data_tx_failure state with probability $1-q$ and $q$, respectively. The mobile moves back to idle/header_tx state once the data packets transmission is complete. Note that the transition from data_tx_failure state to idle/header_tx state is shown in dashed lines to indicate that this transition is possible only in the protocol without LL retransmission, and is not possible in the modified protocol with "persist-until-success" LL retransmission.

If the mobile finds all the uplink channels to be busy upon arrival of a message, it moves from the idle/header_t $x$ state to the backlogged state. Similarly, if the header packet is lost due to collision or bad channel conditions, the mobile moves from the idle/header_tx state to the backlogged state. In the backlogged state, the mobile rechecks the status of the uplink channels after a random number of slots. This rescheduled transmission attempt delay is assumed to be geometrically distributed with parameter $g_{r}$. If a mobile in the backlogged state fails to transmit its header packet successfully, it stays in this state until its header packet transmission is successful, after which it moves to either data_tx_success state or data_tx_failure state.

\section{A. Throughput Performance}

Let $x_{t}$ be the number of mobiles in the data_tx_failure state, $y_{t}$ be the number of mobiles in the data_tx_success state, and $z_{t}$ be the number of mobiles in the backlogged state at the beginning of slot $t$. The three-dimensional (3-D) random process $\left\{x_{t}, y_{t}, z_{t}\right\}$ can be modeled as a finite state Markov chain. For the protocol without LL retransmission, based on the conditional probability that $n$ mobiles simultaneously transmit header packets and $c_{s}$ of those packets are successfully received at the 
base station, the one step transition probability that the system moves from $\left(x_{t}=i_{1}, y_{t}=j_{1}, z_{t}=k_{1}\right)$ at time slot $t$ to $\left(x_{t+1}=i_{2}, y_{t+1}=j_{2}, z_{t+l}=k_{2}\right)$ at time slot $t+1$ is given by

$$
\begin{aligned}
& P_{i_{1} j_{1} k_{1}, i_{2} j_{2} k_{2}} \\
& =\sum_{n=0}^{N-i_{1}-j_{1}} \sum_{c_{s}=0}^{\min \left(M-i_{1}-j_{1}, n\right)} \sum_{s_{j}=0}^{c_{s}} \sum_{s_{f}=0}^{i_{2}-c_{s}+s_{j}} \sum_{f_{s}=0}^{j_{2}-s_{j}} \\
& \times\left(\begin{array}{c}
N-i_{1}-j_{1}-k_{1} \\
a
\end{array}\right) \lambda^{a}(1-\lambda)^{N-i_{1}-j_{1}-k_{1}-a} \\
& \cdot\left(\begin{array}{c}
k_{1} \\
n-a
\end{array}\right) g_{r}^{n-a}\left(1-g_{r}\right)^{k_{1}-n+a} \\
& \cdot\left(\begin{array}{l}
j_{1} \\
b_{j}
\end{array}\right) g_{d}^{j_{1}-b_{j}}\left(1-g_{d}\right)^{b_{j}}\left(\begin{array}{c}
b_{j} \\
s_{f}
\end{array}\right)(1-p)^{s_{f}} p^{b_{j}-s_{f}} \\
& \cdot\left(\begin{array}{l}
i_{1} \\
b_{i}
\end{array}\right) g_{d}^{i_{1}-b_{i}}\left(1-g_{d}\right)^{b_{i}}\left(\begin{array}{c}
b_{i} \\
f_{s}
\end{array}\right)(1-q)^{f_{s}} q^{b_{i}-f_{s}} \\
& \cdot\left(\begin{array}{c}
c_{s} \\
s_{j}
\end{array}\right) p^{s_{j}}(1-p)^{c_{s}-s_{j}} f\left(c_{s} \mid n, M-i_{1}-j_{1}\right)
\end{aligned}
$$

where $M$ is the total number of uplink channels, $N$ is the total number of mobiles, $N \geq M, 0 \leq i_{1} \leq M, 0 \leq j_{1} \leq M-i_{1}$, $0 \leq k_{1} \leq N-i_{1}-j_{1}, 0 \leq i_{2} \leq M, 0 \leq j_{2} \leq M-i_{2}$, $0 \leq k_{2} \leq N-i_{2}-j_{2}, a=k_{2}-k_{1}+c_{s}, b_{j}=j_{2}-s_{j}-f_{s}+s_{f}$, $b_{i}=i_{2}-c_{s}+s_{j}-s_{f}+f_{s}, f_{s} \leq b_{i}, s_{f} \leq b_{j}$, and the conditional probability $f\left(c_{s} \mid n, m_{f}\right)$ is defined as

$$
\begin{aligned}
f\left(c_{s} \mid n, m_{f}\right) & \\
= & \operatorname{Prob}\left(c_{s}\right. \text { header packets are successfully } \\
& \text { received } \mid n \text { mobiles transmit header } \\
& \text { packets over } \left.m_{f} \text { channels }\right) .
\end{aligned}
$$

Note that in (8), the term $\left(\begin{array}{c}N-i_{1}-j_{1}-k_{1} \\ a\end{array}\right) \lambda^{a}(1-\lambda)^{N-i_{1}-j_{1}-k_{1}-a}$ is the probability that $a$ mobiles out of $N-i_{1}-j_{1}-k_{1}$ mobiles in the idle/header_t $x$ state transmit new header packets, $\left(\begin{array}{c}k_{1} \\ n-a\end{array}\right) g_{r}^{n-a}\left(1-g_{r}\right)^{k_{1}-n+a}$ is the probability that $n-a$ mobiles out of $k_{1}$ mobiles in the backlogged state transmit old header packets, $\left(\begin{array}{c}j_{1} \\ b_{j}\end{array}\right) g_{d}^{j_{1}-b_{j}}\left(1-g_{d}\right)^{b_{j}}$ is the probability that $b_{j}$ mobiles out of $j_{1}$ mobiles in the data_tx_success state do not end their message transmissions (since the length of the data segment of the message is geometrically distributed, $g_{d}$ is the probability of ending the message transmission, and $1-g_{d}$ is the probability of continuing with the message transmission), and $\left(\begin{array}{c}b_{j} \\ s_{f}\end{array}\right)(1-p)^{s_{f}} p^{b_{j}-s_{f}}$ is the probability that $s_{f}$ mobiles out of those $b_{j}$ mobiles witness a data packet failure. Also, $\left(\begin{array}{c}i_{1} \\ b_{i}\end{array}\right) g_{d}^{i_{1}-b_{i}}\left(1-g_{d}\right)^{b_{i}}$ is the probability that $b_{i}$ mobiles out of $i_{1}$ mobiles in the data_tx_failure state do not end their message transmissions, $\left(\begin{array}{c}b_{i} \\ f_{s}\end{array}\right)(1-q)^{f_{s}} q^{b_{i}-f_{s}}$ is the probability that $f_{s}$ mobiles out of those $b_{i}$ mobiles witness a data packet success, and $\left(\begin{array}{c}c_{s} \\ s_{j}\end{array}\right) p^{s_{j}}(1-p)^{c_{s}-s_{j}}$ is the probability that $s_{j}$ mobiles out of $c_{s}$ mobiles with successful header packets move to the data_tx_success state. Note that, in the above, we assumed that header packet transmissions do not have any correlation with their previous header or data packet transmissions. That is, header packet success probability is independent of the Markov parameters $p$ and $q$. Also, the probability of moving from header success to data_tx_success is " $p$ " only when there is no capture involved in the header success. However, as an approximation, we use the same " $p$ " as the probability of moving from header capture to data_tx_success. Later, we will see that the numerical results obtained from the analysis using the above approximations closely agree with results obtained from actual simulations.

The conditional probability $f\left(c_{s} \mid n, m_{f}\right)$ can be evaluated by a recursive expression as follows. By conditioning on the number of packets being simultaneously transmitted over an arbitrarily chosen channel (say, the first channel) and using total probability, we have

$$
\begin{aligned}
f\left(c_{s} \mid\right. & \left.n, m_{f}\right) \\
= & \sum_{i=0}^{n}\left(\begin{array}{c}
n \\
i
\end{array}\right)\left(1-\frac{1}{m_{f}}\right)^{n-i}\left(\frac{1}{m_{f}}\right)^{i} \\
& \times f\left(c_{s} \mid n-i, m_{f}-1\right)\left(1-p_{s}^{(i)}\right) \\
& +\sum_{i=0}^{n}\left(\begin{array}{c}
n \\
i
\end{array}\right)\left(1-\frac{1}{m_{f}}\right)^{n-i}\left(\frac{1}{m_{f}}\right)^{i} \\
& \times f\left(c_{s}-1 \mid n-i, m_{f}-1\right) p_{s}^{(i)}
\end{aligned}
$$

where $p_{s}^{(i)}$ is the probability of a header packet capture among $i$ simultaneous transmissions, which is given by [17]

$$
p_{s}^{(i)}=i e^{-1 / F}\left(\frac{1}{1+B}\right)^{i-1}, \quad i \geq 0
$$

where $B$ is the capture threshold. When there is no capture (i.e., $B \rightarrow \infty)$

$$
p_{s}^{(i)}= \begin{cases}1-P_{E}, & i=1 \\ 0, & i>1\end{cases}
$$

The initial conditions for $f\left(c_{s} \mid n, m_{f}\right)$ are given by

$$
\begin{gathered}
f\left(c_{s} \mid n, 0\right)= \begin{cases}1, & \text { if } c_{s}=0 \text { and any } n \\
0, & \text { if } c_{s}>0 \text { and any } n\end{cases} \\
f\left(c_{s} \mid 0, m_{f}\right)= \begin{cases}1, & \text { if } c_{s}=0 \text { and any } m_{f} \\
0, & \text { if } c_{s}>0 \text { and any } m_{f}\end{cases} \\
f\left(c_{s} \mid 1, m_{f}\right)= \begin{cases}1-p_{s}^{(1)}, & \text { if } c_{s}=0 \text { and } m_{f} \geq 1 \\
p_{s}^{(1)}, & \text { if } c_{s}=1 \text { and } m_{f} \geq 1 \\
0, & \text { if } c_{s}>1 \text { and } m_{f} \geq 1\end{cases} \\
f\left(c_{s} \mid n, 1\right)= \begin{cases}1-p_{s}^{(n)}, & \text { if } c_{s}=0 \text { and } n>1 \\
p_{s}^{(n)}, & \text { if } c_{s}=1 \text { and } n>1 \\
0, & \text { if } c_{s}>1 \text { and } n>1\end{cases}
\end{gathered}
$$

and

$$
f\left(c_{s} \mid n, m_{f}\right)=0, \quad \text { if } c_{s}<0 .
$$

If $\mathbf{P}=\left(P_{i_{1} j_{1} k_{1}, i_{2} j_{2} k_{2}}\right)$ is the probability transition matrix and the row vector $\Pi=\left\{\pi_{i_{1} j_{1} k_{1}}\right\}, 0 \leq i_{1} \leq M, 0 \leq j_{1} \leq M-i_{1}$, 
$0 \leq k_{1} \leq N-i_{1}-j_{1}$, contains the steady-state probabilities, then $\Pi$ can be calculated by solving the linear equations $\Pi=$ IIP and using the conservation relationship

$$
\sum_{i_{1}=0}^{M} \sum_{j_{1}=0}^{M-i_{1}} \sum_{k_{1}=0}^{N-i_{1}-j_{1}} \pi_{i_{1} j_{1} k_{1}}=1
$$

The number of successful data packets in a slot is, in this case, equal to the number of users in the data_tx_success state, so that the average number of successes per slot is given by

$$
E\left\{S_{d}\right\}=\sum_{i_{1}=0}^{M} \sum_{j_{1}=0}^{M-i_{1}} \sum_{k_{1}=0}^{N-i_{1}-j_{1}} j_{1} \pi_{i_{1} j_{1} k_{1}}
$$

and the average per channel throughput is obtained as

$$
\eta_{c}=\frac{E\left\{S_{d}\right\}}{M}
$$

\section{B. Delay Performance}

Consider a system containing all mobiles which are either in the backlogged state or in the data_tx_success or data_tx_failure state. The number of users in that system is $\nu=i_{1}+j_{1}+k_{1}$, so that

$$
E\{\nu\}=\sum_{i_{1}=0}^{M} \sum_{j_{1}=0}^{M-i_{1}} \sum_{k_{1}=0}^{N-i_{1}-j_{1}}\left(i_{1}+j_{1}+k_{1}\right) \pi_{i_{1} j_{1} k_{1}} .
$$

On the other hand, the number of users which are not in the system is $N-\nu$, each generating a message in a slot with probability $\lambda$. Whenever a mobile generates a message, it joins the system one slot later. Therefore, the average arrival rate to that system is given by

$$
\Lambda=\lambda(N-E\{\nu\}) .
$$

From Little's formula, the average time which each user spends in the system is given by the ratio between the average number of users in the system and the arrival rate. In our case, the average delay is one slot larger, since a user is assumed to join the system only after the slot in which the message is generated (which is not counted in the above calculation). Therefore, we have for the average delay suffered by a message

$$
E\{D\}=1+\frac{E\{\nu\}}{\Lambda}
$$

\section{LL Retransmission Protocol Performance}

With the "persist-until-success" LL retransmission strategy described in Section II-A, a geometric length message of $X$ packets (with $E\{X\}=1 / g_{d}$ ) will take $X^{\prime}$ slots to finally get through, due to possible LL retransmissions. Therefore, we will have

$$
X^{\prime}=\sum_{i=1}^{X} Y_{i}
$$

where $Y_{i}$ is an integer random variable equal to the number of transmissions it takes data packet $i$ to be successfully received. In the case of i.i.d fading, each packet transmission can experience an error with probability $P_{E}$ and the random variable $Y_{i}$ will have a geometric distribution with parameter $\left(1-P_{E}\right)$. In the case of correlated fading, $Y_{i}$ will have a distribution given by

$$
P\left[Y_{i}=y\right]= \begin{cases}p, & y=1 \\ (1-p) q^{y-2}(1-q), & y \geq 2 .\end{cases}
$$

In both cases, it can be shown that $E\left\{Y_{i}\right\}=1 /\left(1-P_{E}\right)$. Thus, the expected value of the effective length of the message (including the LL retransmission slots) is given by

$$
E\left\{X^{\prime}\right\}=\frac{1}{g_{d}\left(1-P_{E}\right)}
$$

It can be seen that in the protocol with "persist-until-success" LL retransmission, the mobile cannot directly move from the data_tx_failure state to the idle/header_tx state. Thus, the probability term $\left(\begin{array}{c}i_{1} \\ b_{i}\end{array}\right) g_{d}^{i_{1}-b_{i}}\left(1-g_{d}\right)^{b_{i}}\left(\begin{array}{c}b_{i} \\ f_{s}\end{array}\right)(1-q)^{f_{s}} q^{b_{i}-f_{s}}$ in (8) is modified to $\left(\begin{array}{c}i_{1} \\ f_{s}\end{array}\right)(1-q)^{f_{s}} q^{b_{i}-f_{s}}$ and $b_{i}=i_{1}=i_{2}-c_{s}+s_{j}-s_{f}+$ $f_{s}$ in order to account for the fact that, with retransmission of erroneous data packets at the link level, the message transmission cannot end in a data_tx_failure state. Also, the parameter $g_{d}$ in the term $\left(\begin{array}{l}j_{1} \\ b_{j}\end{array}\right) g_{d}^{j_{1}-b_{j}}\left(1-g_{d}\right)^{b_{j}}$ gets replaced with $g_{d}\left(1-P_{E}\right)$.

\section{RESULTS}

Numerical results for the average per channel throughput of the multichannel protocol without LL retransmission, obtained from (20) for $M=3, N=15, g_{d}=0.1, g_{r}=0.1$, and no capture, are plotted in Fig. 2 for a normalized Doppler bandwidth of $f_{D} T=0.02$. The $f_{D} T$ value of 0.02 represents slow fading (i.e., high correlation in fading), and may correspond to the user moving at a speed of $2.5 \mathrm{~km} / \mathrm{h}$ for a carrier frequency of 900 $\mathrm{MHz}$ and a packet duration of $10 \mathrm{~ms}$. The values of the fading margin considered are 5 and $10 \mathrm{~dB}$. Note that at $f_{D} T=0.02$, the fading is slow enough that the channel Markov parameters are the same for threshold, constant $\mathrm{SNR}_{0}$, and constant $P_{E}$ models. The performance in i.i.d. fading is also plotted for comparison. The $g_{d}$ value of 0.1 corresponds to an average message length of ten data packets. Likewise, the parameter $g_{r}=0.1$ means that the average time between rescheduled transmission attempts is ten slots. In addition to the analysis, the multichannel protocol was simulated as well, and the performance collected in over one million slots of simulation runs is also shown in Fig. 2.

From Fig. 2, we observe the following. In i.i.d. fading, when the fading margin is $5 \mathrm{~dB}$, the protocol offers a maximum throughput of about 0.53 . This achievable throughput increases to 0.62 when $f_{D} T=0.02$ (high correlation). Similarly, when $F=10 \mathrm{~dB}$, the i.i.d. fading case yields a maximum throughput of 0.69 , whereas the correlated fading case with $f_{D} T=0.02$ 


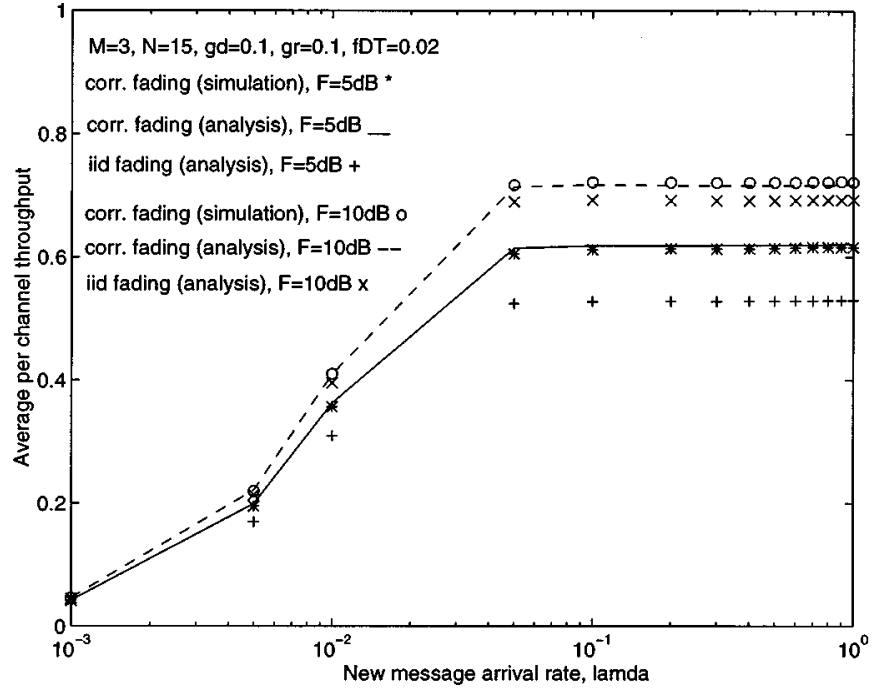

Fig. 2. Average per channel throughput $\eta_{c}$ versus new message arrival rate $\lambda$ in correlated Rayleigh fading. No LL retransmission. $M=3, N=15$, $g_{d}=0.1, g_{r}=0.1, f_{D} T=0.02$, and $F=5$ and $10 \mathrm{~dB}$.

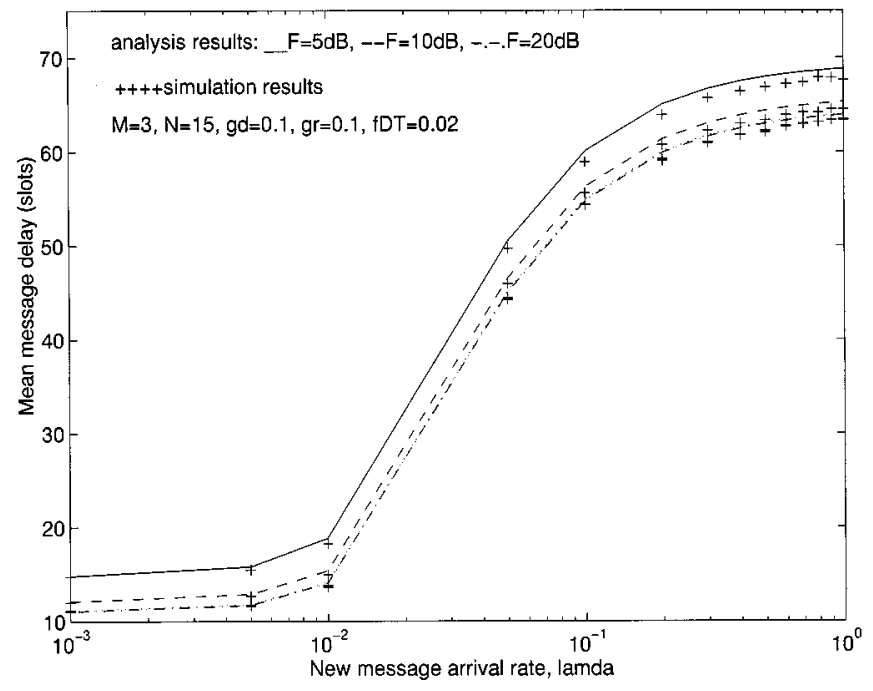

Fig. 3. Mean message delay versus new message arrival rate $\lambda$ in correlated Rayleigh fading. No LL retransmission. $M=3, N=15, g_{d}=0.1, g_{r}=$ $0.1, f_{D} T=0.02$, and $F=5,10$, and $20 \mathrm{~dB}$.

results in a maximum throughput of 0.72 . This shows that the i.i.d. fading model provides a pessimistic performance prediction when the fading is slow (i.e., when there is significant burstiness in packet errors due to high correlation in fading). Also, in Fig. 2, both the analytical and the simulation results for the correlated fading case are found to be in close agreement, thus validating the analysis. Fig. 3 shows the mean message delay performance for the set of parameters in Fig. 2. The curves represent the average delay values, in number of slots, obtained from (23). In Fig. 3, a mean message delay of 11 slots (i.e., $1+1 / g_{d}$ ) at low arrival rates implies that the messages get transmitted immediately on arrival without any waiting/rescheduling delays. Both increased message arrival rates and lower fading margins are seen to increase the mean delay beyond $1+1 / g_{d}$. We also observed from numerical results that the header packet capture phenomenon due to

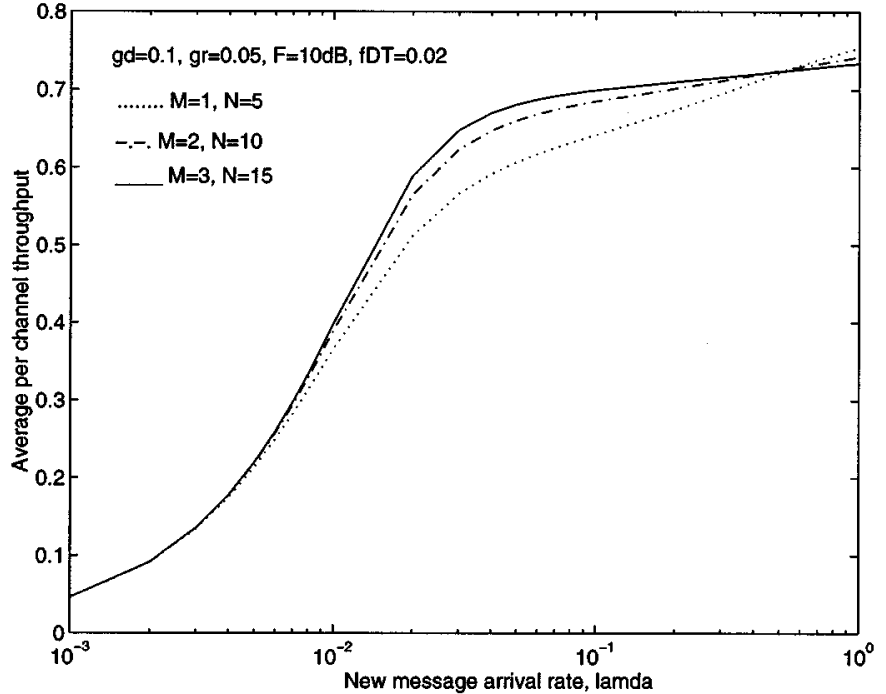

Fig. 4. Effect of number of channels $M$ on the average per channel throughput $\eta_{c}$ for: (a) $M=1, N=5$, (b) $M=2, N=10$, and (c) $M=3, N=15$. No LL retransmission. $g_{d}=0.1, g_{r}=0.05, f_{D} T=0.02$, and $F=10 \mathrm{~dB}$.

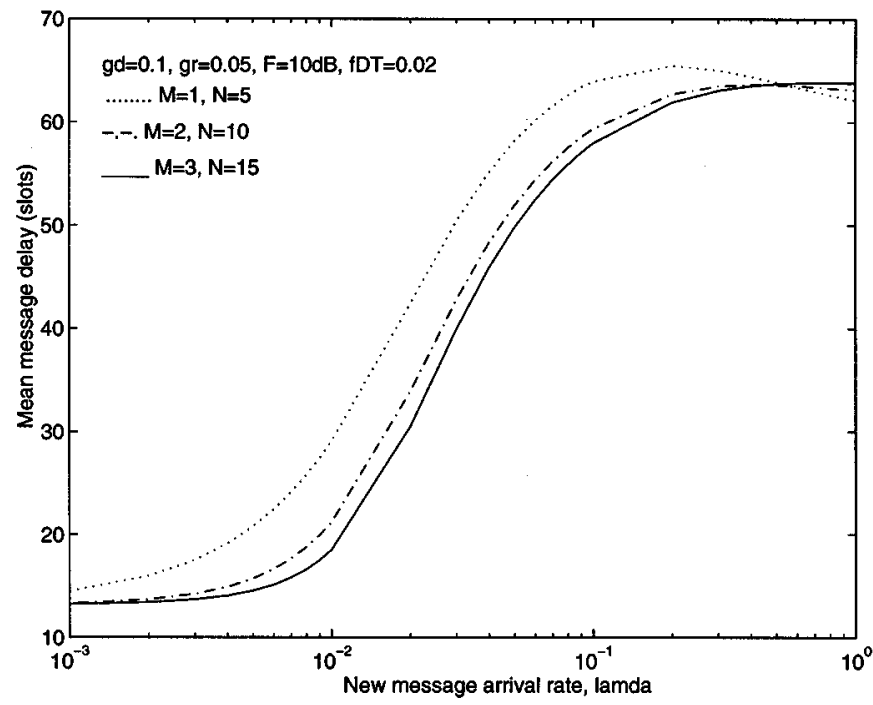

Fig. 5. Effect of number of channels $M$ on the mean message delay for: (a) $M=1, N=5$, (b) $M=2, N=10$, and (c) $M=3, N=15$. No LL retransmission. $g_{d}=0.1, g_{r}=0.05, f_{D} T=0.02$, and $F=10 \mathrm{~dB}$.

multipath fading offers a per channel throughput improvement of about $10 \%-15 \%$, and a similar order of improvement in the delay performance.

The effect of the number of channels $M$ on the throughput and delay characteristics of the multichannel protocol without LL retransmission is shown in Figs. 4 and 5 for $g_{d}=0.1$, $g_{r}=0.05, f_{D} T=0.02, F=10 \mathrm{~dB}$, and no capture. The plots are parameterized by different values of $M$ and $N$, namely: 1) $M=1, N=5$;2) $M=2, N=10$; and 3) $M=3, N=15$, thus keeping the $N / M$ ratio fixed at five in all three cases. For the chosen set of parameters, it is seen that the multichannel system performs better than a single channel system at moderate arrival rates $(0.01<\lambda<0.5)$. This can be explained as follows. If $x$ mobiles $(x>1)$ decide to transmit header packets in a slot, the probability of a success in that slot, assuming no 


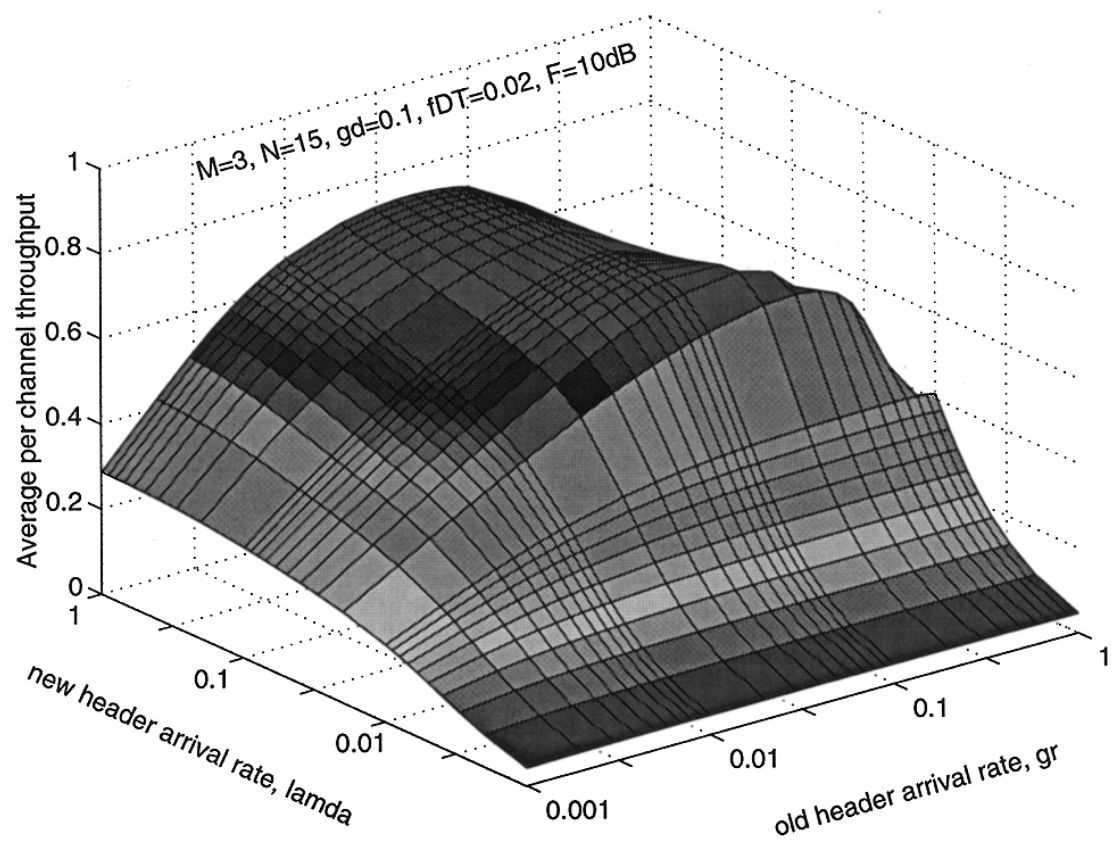

Fig. 6. Effect of $g_{r}$ and $\lambda$ on the average per channel throughput $\eta_{c}$. No LL retransmission. $M=3, N=15, g_{d}=0.1, f_{D} T=0.02$, and $F=10 \mathrm{~dB}$.

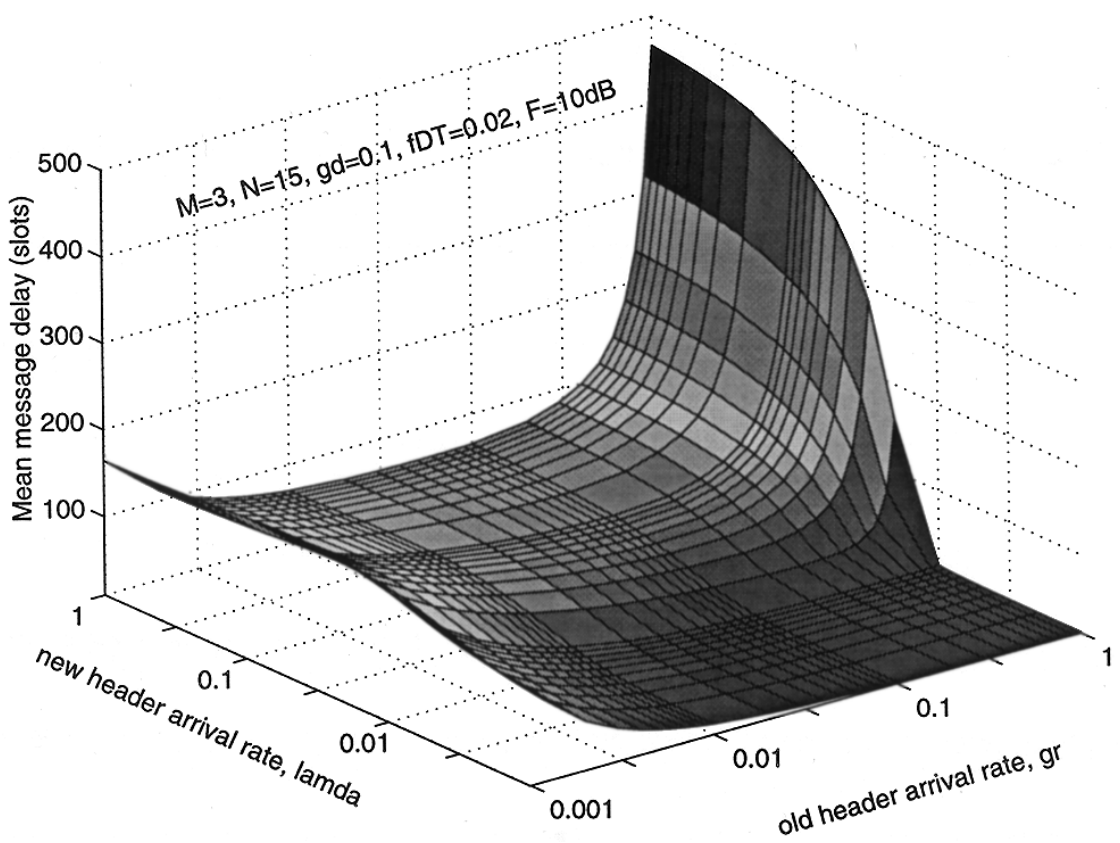

Fig. 7. Effect of $g_{r}$ and $\lambda$ on the mean message delay. No LL retransmission. $M=3, N=15, g_{d}=0.1, f_{D} T=0.02$, and $F=10 \mathrm{~dB}$.

capture, is zero for $M=1$, whereas for $M>1$, the probability of a success will be greater than zero because of the nonzero probability with which any of the $n$ mobiles could pick an idle channel that is contention free from other mobiles. However, at high arrival rates $(\lambda>0.5)$, the multichannel system performs a little poorer than a single channel system. This performance crossover occurs because, at high arrival rates, many of the mobiles could be in the backlogged state, and the number of backlogged mobiles which contend for a slot as soon as a channel becomes idle is higher in the case of $N=15$ than in the case of $N=5$, which increases the probability of a collision. We also observed that increasing the value of $g_{r}$ (i.e., reducing the delay between rescheduled transmission attempts) shifts the crossover point to the left (i.e., toward lower arrival rates).

In Figs. 6 and 7, the effect of the parameter $g_{r}$ on the throughput and delay characteristics of the multichannel protocol without LL retransmission, at various values of new message arrival rate, $\lambda$, is shown as 3 -D contour plots for $M=3, N=15, F=10 \mathrm{~dB}$, and $g_{d}=0.1$. The reason for the bell-shaped curve is that if $g_{r}$ is too small (i.e., more waiting slots in the backlogged state), more slots will go unutilized during the waiting period, and if $g_{r}$ is high, more slots will witness a collision resulting in decreased throughput and increased delays. 


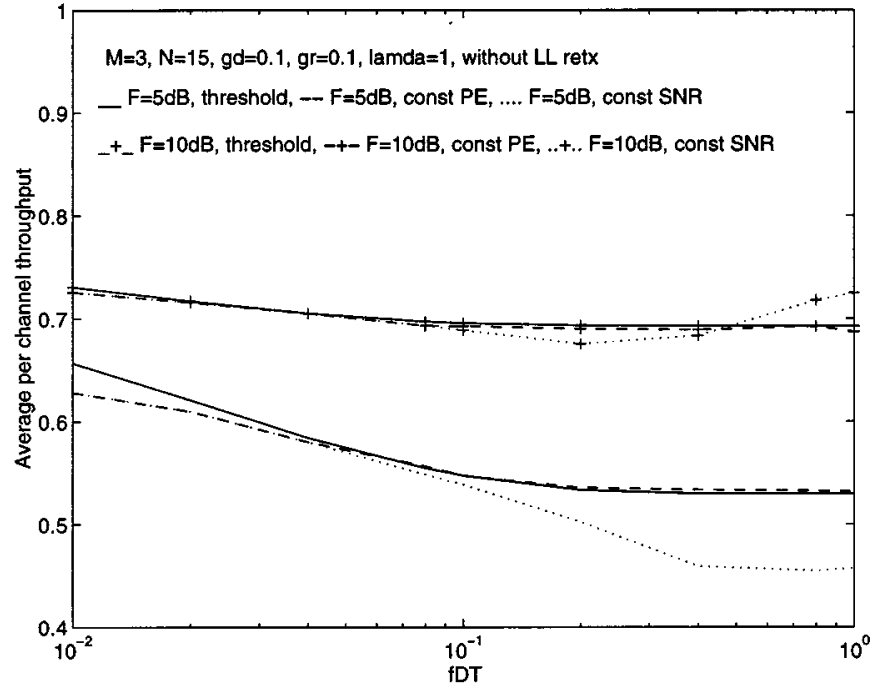

Fig. 8. Average per channel throughput $\eta_{c}$ versus $f_{D} T$. Protocol without LL retransmission. $M=3, N=15, g_{d}=0.1, g_{r}=0.1, \lambda=1$, and $F=5$ and $10 \mathrm{~dB}$.

Fig. 8 shows the throughput performance of the multichannel protocol, without LL retransmission, as a function of the normalized Doppler bandwidth $f_{D} T$ for $\lambda=1, M=3, N=15$, $g_{d}=0.1, g_{r}=0.1, F=5,10 \mathrm{~dB}$, and no capture. The range of $f_{D} T$ values shown corresponds to very slow fading at one end $\left(f_{D} T=0.01\right)$ and close to i.i.d. fading at the other $\left(f_{D} T=1\right)$. The performance achieved using the threshold channel model, constant $\mathrm{SNR}_{0}$ model and constant $P_{E}$ model are compared. As expected, based on the explanation given in Section III, the performance with constant $\mathrm{SNR}_{0}$ and constant $P_{E}$ models coincide for slow fading, as in this case the two models are essentially the same. In this slow fading regime, there is some difference between these results and those obtained from the threshold model, due to the inherent approximations in the latter. On the other hand, as the fading rate increases, the constant $\mathrm{SNR}_{0}$ and constant $P_{E}$ models show slightly different behavior, as the value of $f_{D} T$ affects $P_{E}$ differently in the two cases. It is interesting to see that the constant $P_{E}$ model gives results which are very close to those for the threshold model. It is also interesting to see the behavior of the constant $\mathrm{SNR}_{0}$ case: an increase of the fading rate always degrades the performance for $F=5 \mathrm{~dB}$, whereas it eventually improves it for $F=10 \mathrm{~dB}$. This is explained by noting that in the former case the channel is so bad that the code we are using does not help, and therefore in fast fading more packets are affected by errors which cannot be corrected by the code. On the contrary, as soon as the channel quality is sufficiently good, the joint effect of the error correction code and the time diversity induced by fast fading result in improved packet error rate (and therefore throughput) performance. In any event, it appears that both the the constant $\mathrm{SNR}_{0}$ and constant $P_{E}$ models are fairly well approximated by the threshold model (with the possible exception of the cases in which performance is poor, where it gives an optimistic estimate), which in this case represents a good compromise between accuracy and complexity.

In Figs. 9 and 10, the throughput and delay performance of the multichannel protocol both without LL retransmission and with LL retransmission are compared, as a function of the normalized

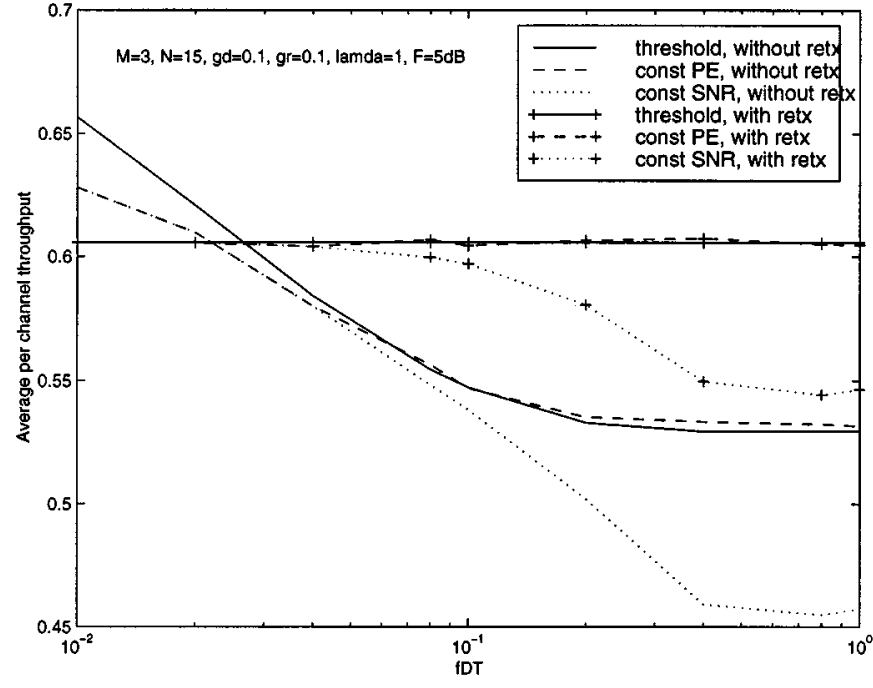

Fig. 9. Average per channel throughput $\eta_{c}$ versus $f_{D} T$. Protocol with and without LL retransmission. $M=3, N=15, g_{d}=0.1, g_{r}=0.1, \lambda=1$, and $F=5 \mathrm{~dB}$.

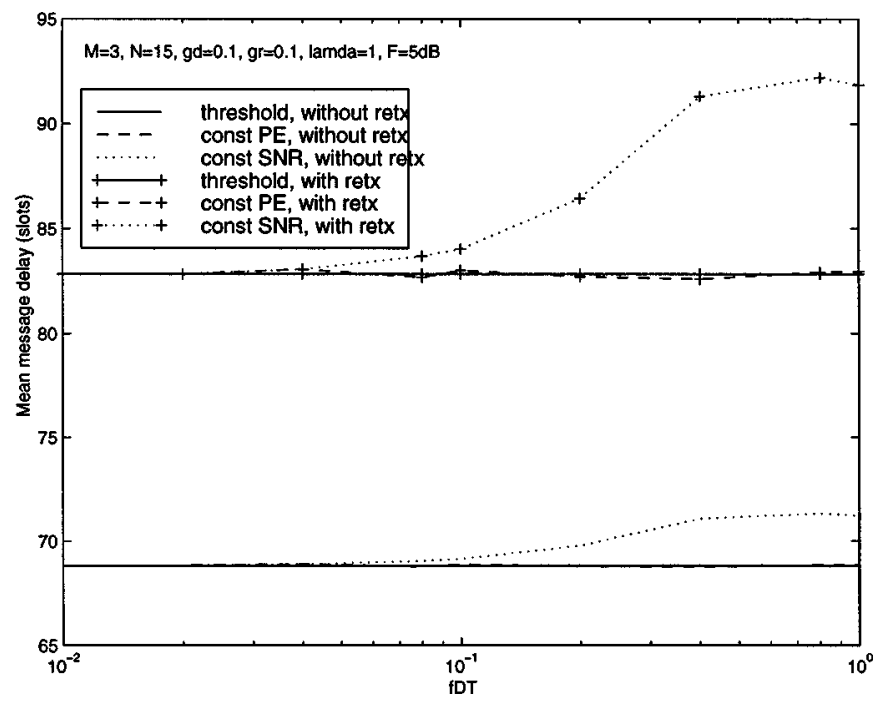

Fig. 10. Mean message delay versus $f_{D} T$. Protocol with and without LL retransmission. $M=3, N=15, g_{d}=0.1, g_{r}=0.1, \lambda=1$, and $F=5$ dB.

Doppler bandwidth $f_{D} T$ for $\lambda=1, M=3, N=15, g_{d}=0.1$, $g_{r}=0.1, F=5 \mathrm{~dB}$, and no capture. When $F=5 \mathrm{~dB}$, the protocol without LL retransmission performs better at low values of $f_{D} T$ compared to large values of $f_{D} T$. The throughput performance of the protocol with LL retransmission remains independent of the value of $f_{D} T$ for the threshold and constant $P_{E}$ models. At low values of $f_{D} T$ (e.g., $f_{D} T<0.02$ ), the protocol without LL retransmission gives better throughput performance than the protocol with LL retransmission, whereas at high values of $f_{D} T\left(f_{D} T>0.02\right)$ the protocol with retransmission LL performs better, consistent with the results presented in [11]. This performance variation over $f_{D} T$ suggests that it is possible to devise more efficient versions of this protocol that could exploit the memory in the channel fading process for better performance. For example, if the base station detects a data packet error from a mobile, it can simply ask the mobile to terminate its ongoing data transmission and release the channel. Such a 
scheme is expected to give good results in the presence of significant channel burstiness (i.e., slow fading), as it avoids insisting on transmission in slots which are likely to be in error, and lets other mobiles (whose channel conditions might be good) to access and use the channel. On the other hand, the above strategy could be wasteful in fast fading conditions where packet errors could occur independently from slot to slot. In such fast fading conditions, error recovery by LL retransmission would be preferred [11].

For the protocol without retransmission, in both the threshold and constant $P_{E}$ models, the delay performance remains the same for both i.i.d. and correlated fading cases (see Fig. 10) because: 1) the average probability of header success $1-P_{E}$ remains independent of the error correlation parameters and 2) since there is no retransmission at the link level, the delay due to data segment transmission is $1 / g_{d}$. We also see that the delay performance remains independent of $f_{D} T$ even in the case of the protocol with "persist-until-success" retransmission. This is because with retransmission, the delay due to data segment transmission is just $1 / g_{d}\left(1-P_{E}\right)$. So both the protocols with and without retransmission will exhibit delay performance independent of $f_{D} T$. However, the protocol with retransmission results in larger delays than the protocol without retransmission. The above observations do not apply to the case of constant $\mathrm{SNR}_{0}$, where the average probability of header success $1-P_{E}$ does depend on $f_{D} T$.

\section{CONCLUSION}

We analyzed the effect of bursty packet losses, caused by correlation in the multipath fading process, on the throughput and delay performance of a multichannel wireless access protocol. $N$ mobile users shared $M$ equal-capacity, orthogonal, traffic channels $(N \geq M)$ on the uplink. Transmission attempts were based on the busy/idle status of the $M$ receivers at the base station. We modeled the fading channel memory using a first-order Markov chain whose parameters were obtained as a function of the normalized Doppler bandwidth. Following a Markov chain analysis, analytical expressions for the average per channel throughput and the mean message delay were derived. Simulation results were shown to verify the analysis. A simple "persist-until-success" retransmission strategy to recover erroneous data packets at the link level was also analyzed. It was shown that the protocol without LL retransmission benefited from highly correlated fading. It was further observed that the multichannel protocol without LL retransmission performed better on slow fading channels (e.g., pedestrian user speeds), whereas the protocol with retransmission performed better in fast fading channels (e.g., vehicular user speeds). It was pointed out, through an example, that more efficient versions of this protocol could be devised which could exploit the memory in the channel fading. The effect of nonzero propagation and processing delays and unreliable feedback on the protocol performance are topics for further investigation.

\section{REFERENCES}

[1] K. Pahlavan and A. H. Levesque, Wireless Information Networks. New York: Wiley, 1995
[2] W.-C. Wong, "Packet reservation multiple access in a metropolitan microcellular radio environment," IEEE J. Select. Areas Commun., vol. 11, pp. 918-925, Aug. 1993.

[3] N. D. Wilson, R. Ganesh, K. Joseph, and D. Raychaudhuri, "Packet CDMA versus dynamic TDMA for multiple access in an integrated voice/data PCN," IEEE J. Select. Areas Commun., vol. 11, pp. 870-884, 1993.

[4] Z. Liu and M. E. Zarki, "Performance analysis of DS-CDMA with slotted ALOHA random access for packet PCNs," Wireless Networks, vol. 1, no. 1, pp. 1-16, 1995.

[5] F. Borgonovo, M. Zorzi, L. Fratta, V. Trecordi, and G. Bianchi, "The capture-division packet access (CDPA) for wireless personal communications," IEEE J. Select. Areas Commun., vol. 14, pp. 609-623, May 1996.

[6] X. Qiu and V. O. K. Li, "Dynamic reservation multiple access (DRMA): A new multiple access scheme for personal communication system," Wireless Networks, pp. 117-128, June 1996.

[7] W. Yue, "The effect of capture on performance of multichannel slotted ALOHA systems," IEEE Trans. Commun., vol. 39, pp. 818-822, June 1991.

[8] Z. Zhang and Y.-J. Liu, "Comments on the effect of capture on performance of multichannel slotted ALOHA systems," IEEE Trans. Commun., vol. 41, pp. 1433-1435, Oct. 1993.

[9] R. H. Clarke, "A statistical theory of mobile radio reception," Bell Syst. Tech. J., vol. 47, pp. 957-1000, July 1968.

[10] W. C. Jakes, Jr., Microwave Mobile Communications. New York: Wiley, 1974.

[11] A. Chockalingam, M. Zorzi, L. B. Milstein, and P. Venkataram, "Performance of a wireless access protocol on a correlated Rayleigh fading channel with capture," IEEE Trans. Commun., vol. 46, pp. 644-655, May 1998.

[12] D. Bertsekas and R. Gallager, Data Networks. Englewood Cliffs, NJ: Prentice-Hall, 1987.

[13] W. Xu, A. Chockalingam, and L. B. Milstein, "Throughput-delay analysis of a multichannel packet CDMA scheme in a fading environment," in Proc. IEEE ICUPC'97, vol. 1, San Diego, CA, Oct. 1997, pp. $183-187$.

[14] M. Zorzi, R. R. Rao, and L. B. Milstein, "On the accuracy of a first-order Markov model for data transmission on fading channels," in Proc. IEEE ICUPC'95, Nov. 1995, pp. 211-215.

[15] _ _ "Error statistics in data transmission over fading channels," IEEE Trans. Commun., vol. 46, pp. 1468-1477, 1998.

[16] S. Lin and D. J. Costello, Jr., Error Control Coding: Fundamentals and Applications. Englewood Cliffs, NJ: Prentice-Hall, 1983.

[17] M. Zorzi and R. R. Rao, "Capture and retransmission control in mobile radio," IEEE J. Select. Areas Commun., vol. 12, pp. 1289-1298, Oct. 1994.

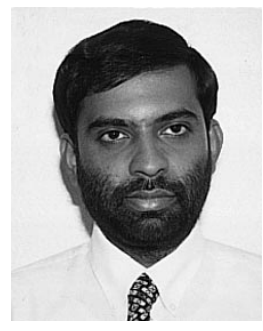

A. Chockalingam (S'92-M'95-SM'98) was born in Rajapalayam, Tamilnadu State, India. He received the B.E. degree in electronics and communication engineering (Honors) from the P.S.G. College of Technology, Coimbatore, India, in 1984, the M.Tech. degree with specialization in satellite communications from the Indian Institute of Technology, Kharagpur, India, in 1985, and the Ph.D. degree in electrical communication engineering from the Indian Institute of Science (IISc.), Bangalore, India, in 1993.

From 1986 to 1993, he was with the Transmission R\&D Division of the Indian Telephone Industries Ltd., Bangalore. From December 1993 to May 1996, he was a Post-Doctoral Fellow and an Assistant Project Scientist at the Department of Electrical and Computer Engineering, University of California, San Diego (UCSD), where he conducted research in DS-CDMA wireless communications. From May 1996 to December 1998, he was with Qualcomm, Inc., San Diego, as a Staff Engineer/Manager in the Systems Engineering Group. In December 1998, he joined the faculty of the Department of Electrical Communication Engineering, IISc., where he is an Assistant Professor working in the area of wireless communications and directing research at the Wireless Research Lab (WRL), IISc. He was a Visiting Faculty Member at UCSD during the summer of 1999. His research interests lie in the area of DS-CDMA systems and wireless networks and protocols.

Dr. Chockalingam was the recipient of the CDIL (Communication Devices India Ltd.) Award for a paper published in the IETE Journal. 


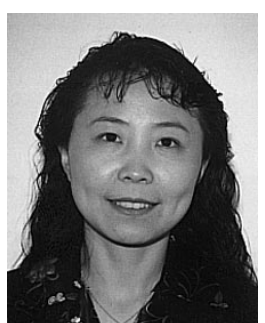

Weiping Xu (M'98) received the B.S. and M.S. degrees in radio engineering from Southeast University, China, in 1985 and 1988, respectively, and the Ph.D. degree in electrical engineering from the University of California, San Diego, in 1998.

She is currently a Member of Technical Staff with the Research and Development Center at Nokia Mobile Phones, San Diego, specializing in communication system design. From 1988 to 1995 , she was on the faculty of the National Mobile Communication Research Laboratory at Southeast University. She was a Communication System Engineer at Hung Nien Electronics, Hong Kong, from 1991 to 1993. Her research interests include digital communication theory with special emphasis on spread-spectrum communication systems and multimedia access protocols.

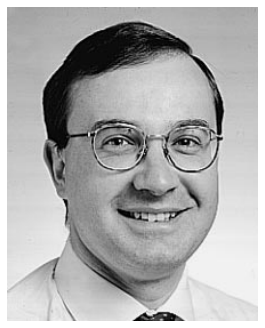

Michele Zorzi (S'89-M'95-SM'98) was born in Venice, Italy, in 1966. He received the Laurea and $\mathrm{Ph} . \mathrm{D}$. degrees in electrical engineering from the University of Padova, Padova, Italy, in 1990 and 1994, respectively.

From 1992 to 1993, he was on leave at the University of California, San Diego (UCSD), attending graduate courses and doing research on multiple access in mobile radio networks. In 1993, he joined the faculty of the Dipartimento di Elettronica e Informazione, Politecnico di Milano, Milan, Italy. After spending three years with the Center for Wireless Communications at UCSD, in 1998 he joined the School of Engineering at the Università di Ferrara, Ferrara, Italy, where he is currently an Associate Professor. His present research interests include performance evaluation in mobile communications systems, random access in mobile radio networks, and energy-constrained communications protocols. He currently serves on the Editorial Board of the IEEE Personal Communications Magazine. He is also a Guest Editor in the area of Energy Management in Personal Communications Systems for special issues of the IEEE Personal Communications Magazine and the IEEE JOURNAL ON SELECTED AREAS IN COMMUNICATIONS.

Dr. Zorzi serves on the Editorial Board of the ACM/URSI/Baltzer Journal of Wireless Networks in the area of Multimedia Network Radios.

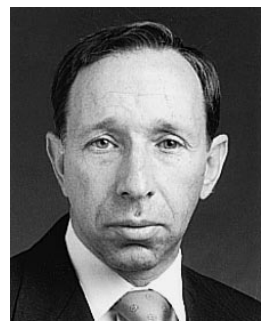

Laurence B. Milstein (S'66-M'68-SM'75-F'85) received the B.E.E. degree from the City College of New York in 1964 and the M.S. and Ph.D. degrees in electrical engineering from the Polytechnic Institute of Brooklyn, Brooklyn, NY, in 1966 and 1968, respectively.

From 1968 to 1974, he was with the Space and Communications Group of Hughes Aircraft Company, and from 1974 to 1976, he was a Member of the Department of Electrical and Systems Engineering, Rensselaer Polytechnic Institute, Troy, NY. Since 1976, he has been with the Department of Electrical and Computer Engineering, University of California at San Diego, La Jolla, CA, where he is a Professor and former Department Chairman working in the area of digital communication theory with special emphasis on spread-spectrum communication systems. He has also been a consultant to both government and industry in the areas of radar and communications. He was an Associate Editor of Communication Theory for the IEEE TRANSACTIONS ON COMMUNICATIONS, an Associate Editor of Book Reviews for the IEEE TRANSACTIONS ON INFORMATION THEORY, an Associate Technical Editor for the IEEE COMMUNICATIONS MAGAZINE, and the Editor-in-Chief of the IEEE JOURNAL ON SELECTED AREAS IN COMMUNICATIONS.

Dr. Milstein was the Vice President of Technical Affairs in 1990 and 1991 for the IEEE Communications Society and has been a member of the Board of Governors for both the IEEE Communications Society and the IEEE Information Theory Society. He is also a member of Eta Kappa Nu and Tau Beta Pi. He was the recipient of the 1998 IEEE MILCOM Long Term Technical Achievement Award. 